\title{
Authentication System Using Location Information on iPad or Smartphone
}

\author{
Hideyuki Takamizawa and Noriko Tanaka
}

\begin{abstract}
In the mobile computing industry, smartphones and iPads have emerged as realistic substitutes for notebook computers. For example, the iPad is designed to support a variety of media, including video, sound, and websites. It is different from a cell phone in that it has the ability to use rich e-learning content. However, the iPad does not include special structures for user authentication. For example, it does not have a camera or a device for fingerprint authentication. The purpose of this paper is to explain how to perform effective personal authentication using the built-in capabilities of the iPad or smartphone. For this purpose, we developed a prototype of the authentication system using location information. Fortunately, the iPad and many smartphones came equipped with a GPS facility that can acquire positional information. We compared the information provided by the known address block with the information provided by the iPad (latitude and longitude coordinates). The test results indicate that the developed prototype is effective.
\end{abstract}

Index Terms-Smartphone, authentication, GPS

\section{INTRODUCTION}

In asynchronous mobile learning, it is difficult for a teacher to authenticate a student. The most common authentication approach is to use an ID-password pair. ID-password authentication is well established, mainly because it is easy to implement and use. However, students who ask their friends to complete their assignments may give them their ID-password pair; therefore, this authentication method cannot effectively prevent identity theft or abuse. In recent years, authentication that uses the cell phone number or SIM card number has become more popular. We proposed a method whereby a cell phone is used as an authentication token in web-based education [1]. Other researchers have proposed authentication method using multiple communication channels [2]-[4]. In a simple example, authentication is performed with a service coupon or member's card using the cell phone number. This method is effective in iPads or smartphones. However, it cannot guard against a person who purchases a cell phone simply for authentication purposes, perhaps with the intention of a spoofing attack. De Cock [5] insists that the very owner of the security token is a security risk because it is easy to give away the token.

In computer systems, authentication refers to the identification of an individual using attributes that only the

Manuscript received February 16, 2012; revised March 28, 2012.

Hideyuki Takamizawa is with the Center for Information and Technology, Hitotsubashi University, Japan (e-mail: jetta@w5.dion.ne.jp).

Noriko Tanaka is with the Center for Information and Media, Rissyo University, Japan. identified person "knows" (e.g., passwords, PINs), "has" (e.g., tokens, smart cards), or "is" (e.g., fingerprint, face). More recently, it has also become possible to use the information "where you are" via GPS positional information.

For example, Facebook provides a service called "Places" (Fig. 1), which includes positional information for the IOS and Android mobile operating systems [6]. Facebook users can share "where you are". For example, using "Places," a user can contact a friend who is nearby and exchange information about a shop in the vicinity. "Places" is a typical example of a service that uses positional information.

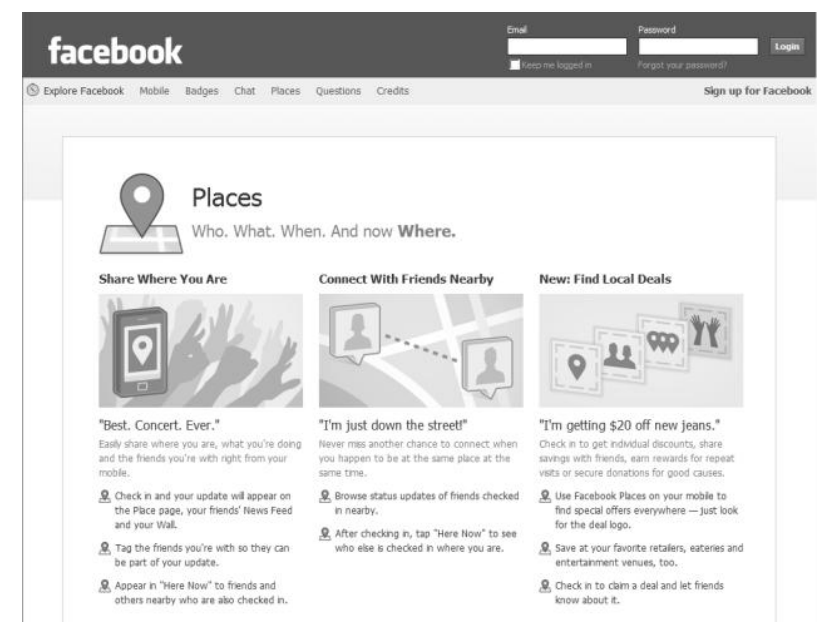

Fig. 1. Magnetization as a function of applied field

In other examples, Y. Zheng developed a model based on the user's transportation mode (e.g., walking, driving, etc.), including keeping track of changes in positional information using a GPS [7]. In addition, we have previously suggested an authentication method using positional information on a personal computer [8]. Although there have been many studies on possible services that use positional information, very few have investigated the use of the relationship between the positional information and a physical address. J. Hightower and G. Borriello [9] suggested using location information in ubiquitous computing, highlighting the accuracy and limitations of using GPS in this way. However, their subject is more technical in nature, and does not easily lend itself to real-world applications.

In our study, we built a practical prototype that supports iPads and smartphones, and enables these devices to acquire positional information from an address and from GPS positional information. The experiment was conducted using more than 40 addresses. We found that the positional information we obtained from the iPad or smartphone, along with relevant error information, was sufficient to pinpoint those addresses.

In Section II, we explain the proposed authentication 
method. In Section III, we explain the experimental methodology and present the results. Section IV concludes the paper and discusses directions for future study.

\section{MODEL}

\section{A. What Kind of Learning Model is This Paper aimed at?}

E-learning has many learning models. In our model, we present a method that identifies a person in a distance learning environment. In distance learning, students can access the courses and examinations from anywhere, at any time. However, our method uses personal identification to limit where a student can access an examination.

When a student takes an examination, authentication is necessary because the examination is directly related to the evaluation of the student. However, when a student learns coursework, the strength of the authentication need not be at the same level. In this case, no authentication or simple authentication is often sufficient. We do not want to deprive students of the opportunity to learn a course anywhere and anytime.

\section{B. Obtaining Location Information from an iPad or Smartphone}

The iPad and many smartphones come equipped with a GPS facility that can acquire positional information. Location information for a cell phone is provided in the form of latitude and longitude values by a special parameter in the web-access functionality on the phone.

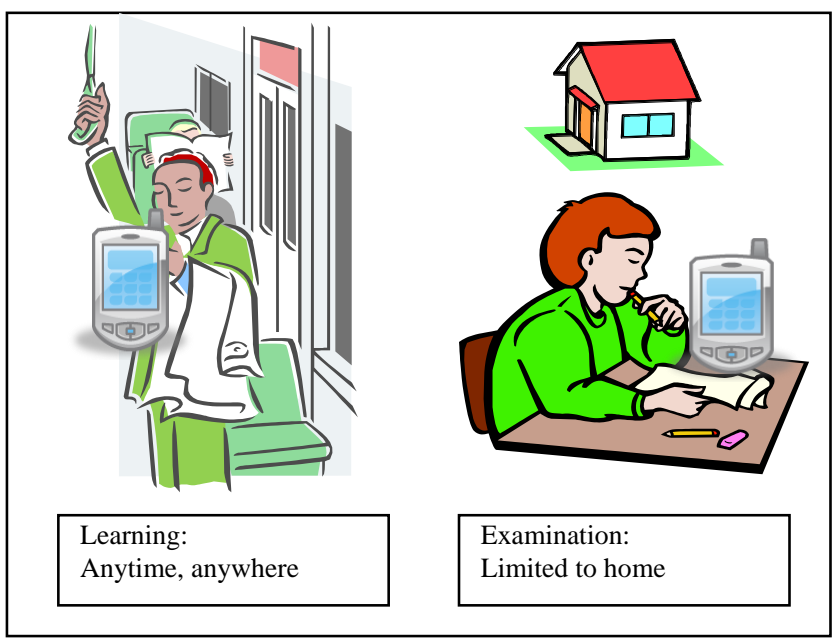

Fig. 2. Proposed distance learning model

These parameters differ according to the specifications of the cell phone carrier. In the case of an iPad and a smartphone, we can use the Geolocation API in an operating system and browser combination, such as iOS and Safari, or Android and Chrome Lite [10]. The programming code used to acquire a position is very simple. Fig. 3 shows a sample of the JavaScript code.

This sample code provides latitude, longitude, and precision information. Note that the code uses a program called "database.asp" to acquire the location information.

\section{Latitude and Longitude Information and the Address}

In general, the educational institution knows the address of the student. However, the student's address is not stated in terms of latitude and longitude information. Therefore, we need to convert the student's physical address into latitude and longitude information to verify that a student is in his or her home.

There are many methods for converting an address into location information, but a simple method, available in many countries, uses the Google MAPS API [11], [12]. Fig. 4 shows the code to convert a physical address into latitude and longitude values using the Google MAPS API. Although we converted the Japanese address in Japan, of course, Google MAPS API corresponds to many languages.

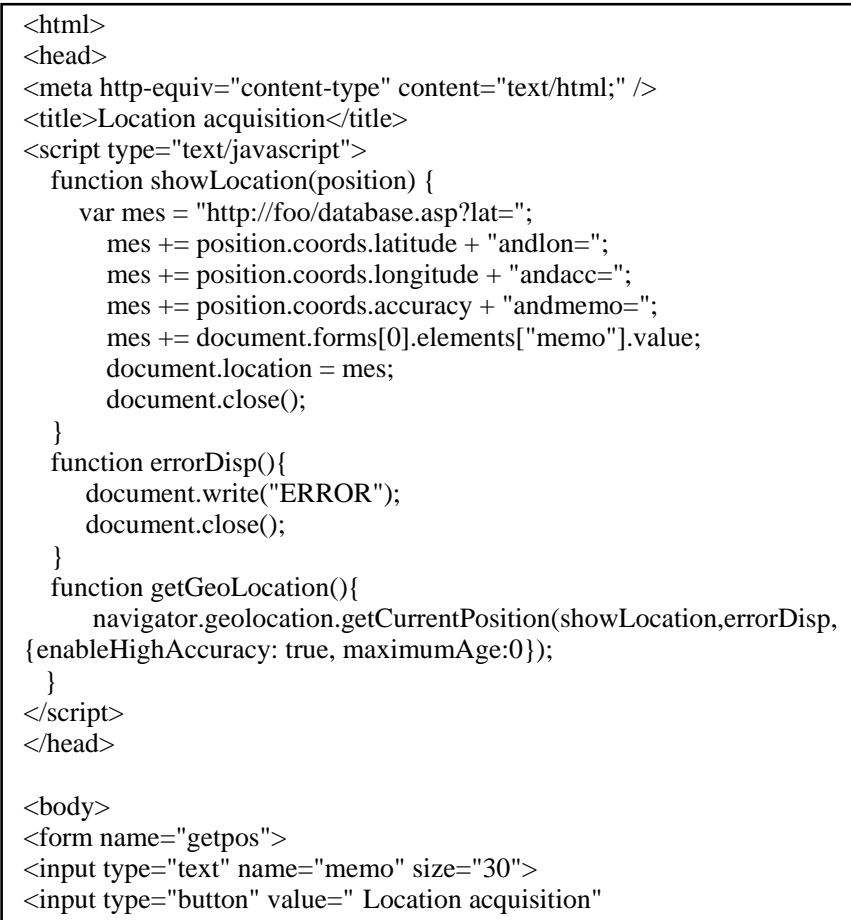

Fig. 3. JavaScript code to acquire location.

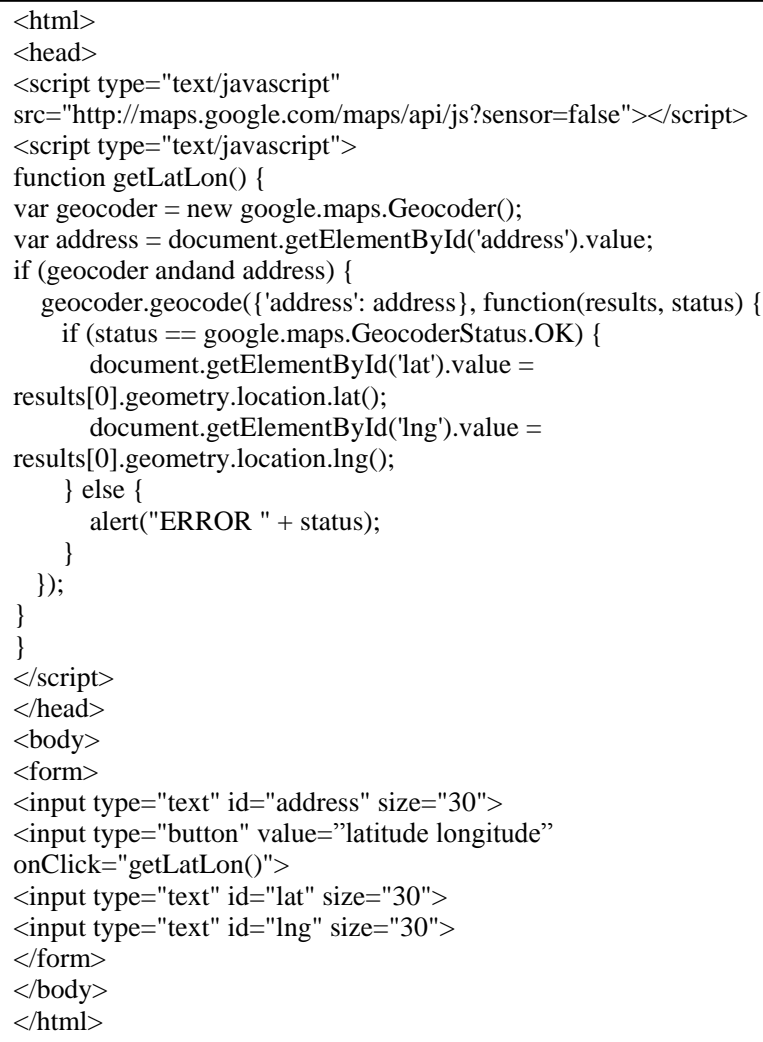

Fig. 4. Acquiring latitude and longitude information. 


\section{Identifying of the Location of the Examination}

The latitude and longitude values provided by the GPS indicate the center of a circle. The circle is as accurate as the GPS can be. Therefore, there is a potential error in the coordinates. However, if the student's address appears within that circle, we can be reasonably sure that the student is at home (see Fig. 5).

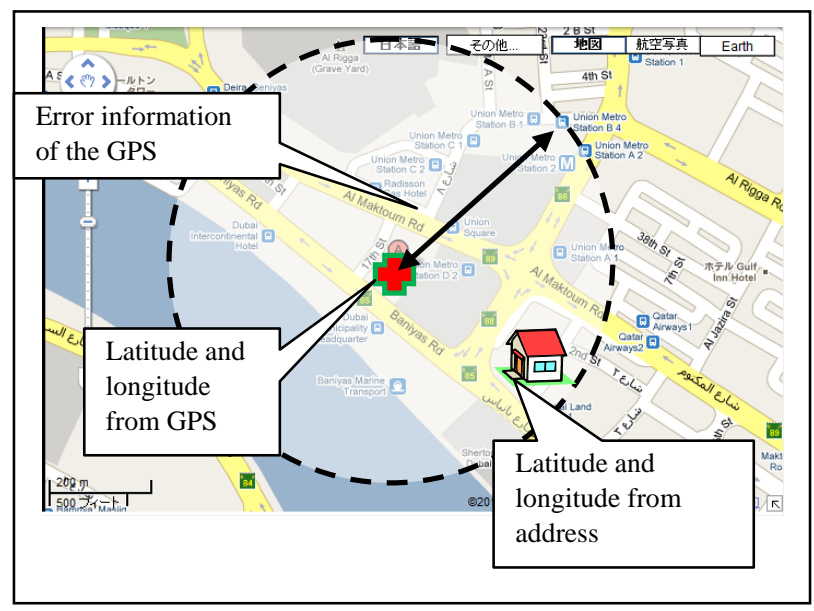

Fig. 5. Position relations.

We can use Hybeny's distance formula [13] (shown in Fig. 6.) to calculate the distance between the latitude and longitude values of the GPS coordinates and those of the address.

$\mathrm{D}=\sqrt{(\mathrm{M} \times \mathrm{dP})^{2}+(\mathrm{N} \times \cos (\mathrm{P}) \times \mathrm{dR})^{2}}$

D: Distance between two points (m)

$\mathrm{P}$ : Average latitude of two points

dP: Latitude difference between two points

dR: Longitude difference between two points

M: Radius of meridian curvature

$\mathrm{N}$ : Radius of prime vertical curvature

Fig. 6. Hybeny's distance formula.

We do not have to know the position of the examinee precisely. The address of the examinee must simply exist within the error range of the GPS. The purpose of this method is to prevent another person from pretending to be the examinee.

In distance learning, spoofing is possible from anywhere in the world. However, the possibility that a neighbor performs spoofing is very low. Therefore, even though there is a possibility of error, we can be far more certain that the correct person is taking the examination.

\section{EXPERIMENT}

\section{A. Purpose of the Experiments}

The purpose of the experiments is to confirm that this authentication model functions adequately. In general, students take distance learning examinations indoors, so here we measure positional information and error information with a smartphone inside a building. We can say that the authentication model works if the location information obtained from the student's address is in the error range of the

\section{GPS coordinates.}

\section{B. Simulation of the Learning Environment}

We chose a convenience store as a simulated learning environment. The reasons are as follows:

- The store exists in many places.

- The store address is available on the Internet.

- The measurement that uses GPS with a smartphone is inside the building.

- The area of the shop is approximately equal to that of a house.

- A structure such as a wall or window is similar to that of a house.

We acquired the location information by using an iPad in the experiment, and using the $3 \mathrm{G}$ network and GPS in the store. Fig. 7 shows an external view of the convenience store.

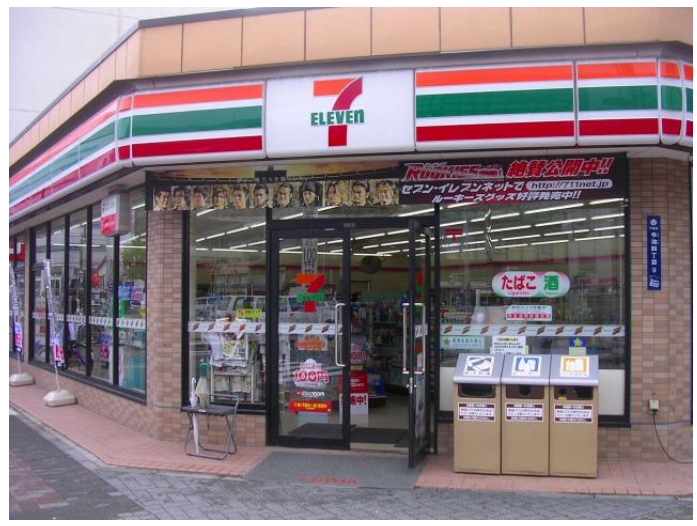

Fig. 7. Convenience store used in the experiment

\section{Experiment locations}

We carried the experiment in the suburbs of Tokyo, including the location of 46 convenience stores. Fig. 8 shows a map of the area. The pins show the convenience stores.

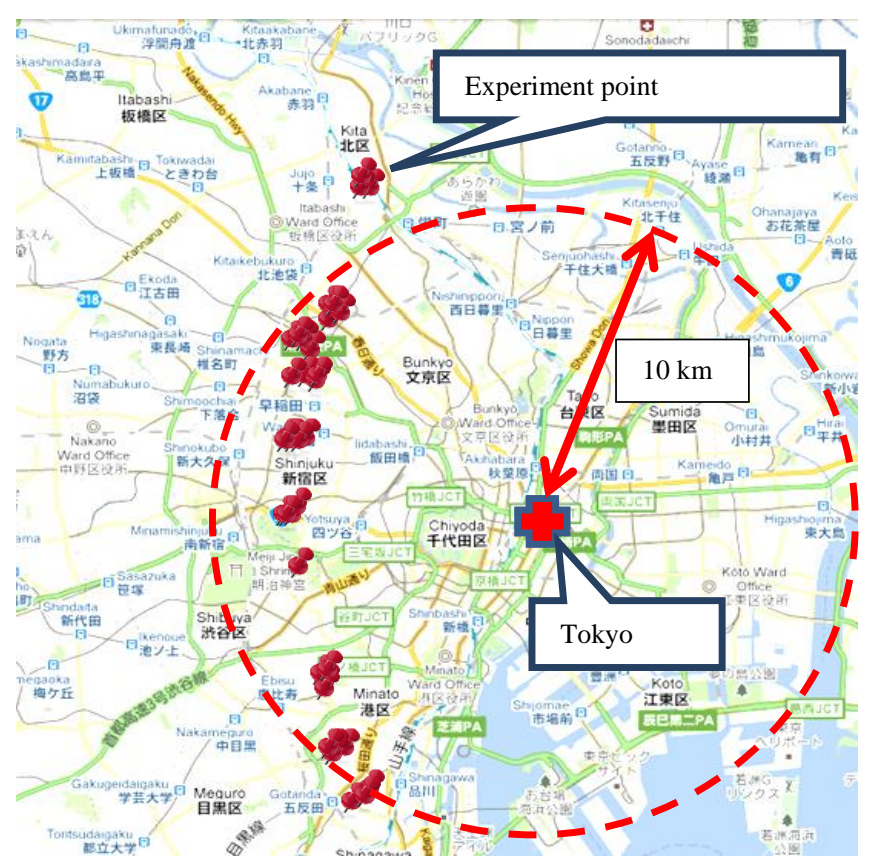

Fig. 8. Map of place

\section{Results}

The results of the experiment are summarized in two scatter diagrams. Fig. 9 shows the difference between the "Location obtained from the address" and the "Location 
obtained from GPS". Fig. 10 shows the ratio of the error margin. The dotted line showing $100 \%$ is the error range of the GPS.

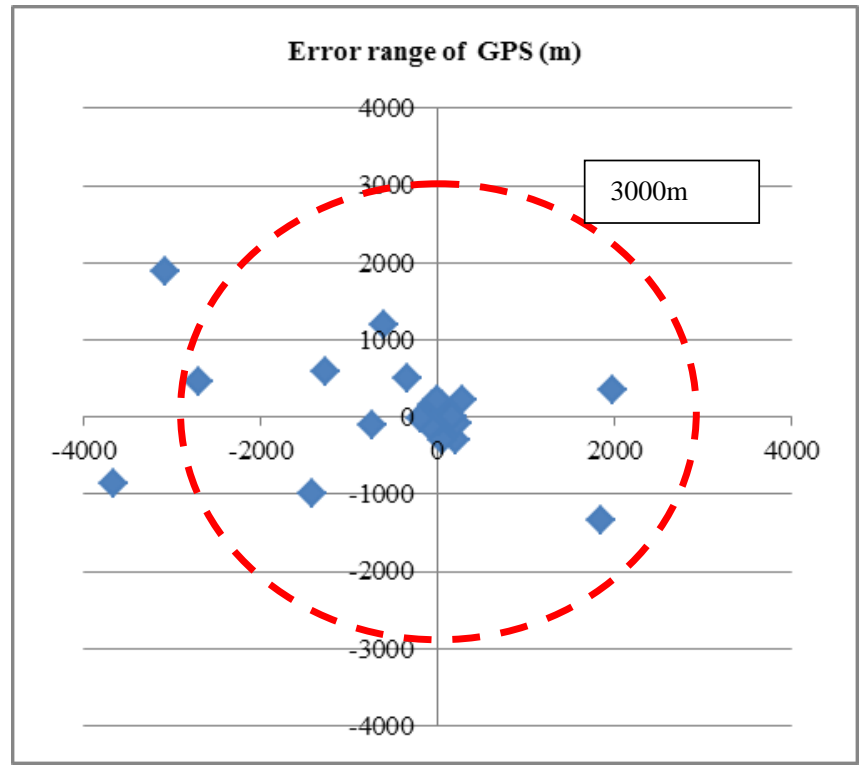

Fig. 9. Error range of GPS (m)

Most of the data falls within an error range of less than $3000 \mathrm{~m}$. The mean of the errors is $1631.1 \mathrm{~m}$. However, this precision is worse than that of the cell phone GPS. The conditions are not identical; however, according to our previous experiment [8], the precision of the cell phone GPS has an average of $142.76 \mathrm{~m}$.

Most of the data falls within the error range of the GPS. The data values that fell outside the error range were not far outside, for example, $5 \mathrm{~m}$ and $30 \mathrm{~m}$ outside the error range.

An error value of the GPS has to consider the size of the block that the address identifies. In general, the size of the block of an address is the same as the size of the house. However, in a rural area, the size of the address block becomes bigger.

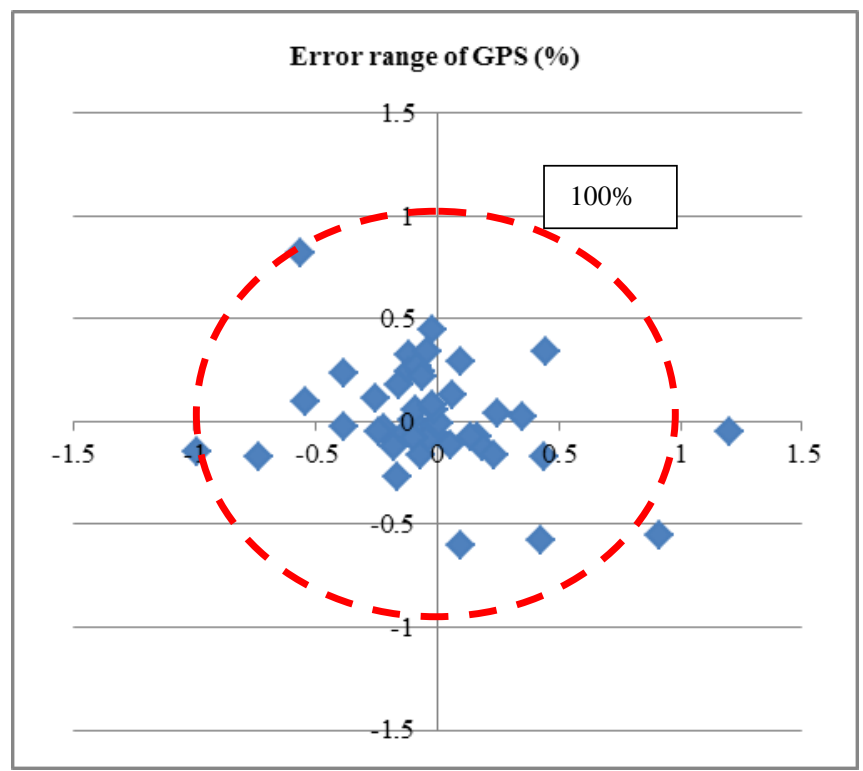

Fig. 10. Error range of GPS (\%)

\section{CONCLUSION}

In distance learning, it is difficult to identify students because web-based distance learning is asynchronous, and teachers cannot authenticate students directly. In this paper, we have proposed a new authentication method that uses location information, and we have shown how to use it by implementing a prototype.

Next, we investigated the reliability of the location information from an iPad or smartphone. We simulated the learner's environment by using a convenience store. Our assumption is that the learner engages with web-based learning at a location with a known address (latitude and longitude). From our results, we concluded that the location information from the iPad or smartphone provides enough information to support authentication.

However, in order to enhance the scope of our system, the algorithm that verifies the student's location needs to consider the size of the address block.

When a higher-education institution offers credits via web-based education, it is very important that the institution counters academic dishonesty. The proposed method requires further development, but it can provide a potential solution.

\section{REFERENCES}

[1] H. Takamizawa and K. Kaijiri, "Reliable authentication method by using cellular phones in web based training", International Journal of Instructional Technology and Distance Learning, vol. 3, no. 12, 2006, pp. 35-46, December 2006.

[2] S. Mizuno, K.Yamada, and K.Takahashi, "Authentication using multiple communication channels", DIM '05 Proceedings of the 2005 workshop on Digital identity management, pp. 54-62, January 2006.

[3] Min Wu, S. Garfinkel, and R. Milleret, "Secure web authentication with mobile phones", MIT Computer Science and Artificial Intelligence Laboratory, Student Oxygen Workshop 2003.

[4] M. Mannan and P. C. Van Oorschot, "Using a personal device to strengthen password authentication from an untrusted computer", FC'07/USEC'07 Proceedings of the 11th International Conference on Financial cryptography and 1st International conference on Usable Security, pp. 88-103, February 2007.

[5] D. De Cock, K. Wouters, D. Schellekens, D. Singelee, and B. Preneel, "Threat modelling for security tokens in web applications", 8th IFIP Conf. on Communications and Multimedia Security, pp. 183-193, Vienna, 1994.

[6] Facebook Official Site, "Places - Who. What. When. And now Where", http://www.facebook.com/places/, Internet, retrieved November 2010.

[7] Y. Zheng, L. Liu, L.Wang, and X. Xie, "Learning transportation mode from raw GPS data for geographic applications on the web", WWW '08 Proceeding of the 17th international conference on World Wide Web, Beijing, pp. 247-256, April 2008.

[8] H. Takamizawa and K. Kenji, "A web authentication system using location information from mobile telephones", Proceedings of the IASTED international conference Web-based Education (WBE 2009), Phuket, pp 31-36, March 2009.

[9] J. Hightower and G. Borriello, "Location systems for ubiquitous computing", Computer, vol. 34, no. 8, pp. 57-66, 2001.

[10] W3C Editor's Draft, "Geolocation API specification", http://dev.w3.org/geo/api/spec-source.html, Internet, retrieved November 2010.

[11] Google code, "The Google Geocoding API (V3)" http://code.google.com/intl/en/apis/maps/documentation/geocoding/, Internet, retrieved November 2010

[12] Hirafoo, "Geocoding in Google maps API (in Japanese)", http://d.hatena.ne.jp/hirafoo/20100911/1284135763, Internet, retrieved November 2010.

[13] M. Nakashiki, H. Narita, and Y. Higashiyama, "Appropriateness of inexpensive hand-held global positioning system units for creating weed maps in a pasture", Weed Biology and Management, vol. 6, no. 4, pp. 183-188, 2006. 


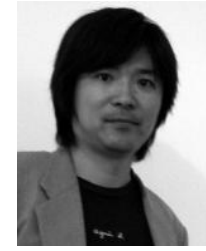

Hideyuki Takamizawa, Ph.D. Associate Professor, Center for Information and Technology, Hitotsubashi University, Japan. He has received B.A. from The Open University of Japan, in 2002, and M.E., and Ph.D. in Engineering degrees from Shunshu University, Japan, in 2004, and 2007. His areas of research interest are software engineering and distance learning. He is a member of the IEEE.
Noriko Tanaka, Technical Staff, Center for Information and Media, Rissyo University, Japan. She has received B.L. from The Rissyo University of Japan, in 1999. Her area of research interest is distance learning. 